\title{
ANTECEDENTES MEDIEVALES DE LOS DERECHOS HUMANOS
}

Jorge M. Ayala*

SÍNTESIS - Una de las mayores revoluciones de la época contemporánea en el plano jurídicomoral es la universalización de los derechos humanos. Esta nueva realidad que vive la Humanidad al término del II Milenio es fruto de una larga conquista colectiva, pues todas las civilizaciones se fundan en sistemas de creencias que tratan de liberar al hombre de las necesidades más elementales de la condición humana. Sin embargo, han sido la cultura occidental la que más se ha distinguido en la lucha por las libertades, como ha quedado recogido en la literatura del Antiguo y del Nuevo Testamento y en las de los griegos y romanos. Ciertamente no se trata aún de los derechos subjetivos, pero la idea de naturaleza humana inspira los primeros intentos de elevar al plano jurídico y político los deseos legítimos de libertad. En el siglo XII surgen las primeras ciudades "libres" y se reconoce la iniciativa de mercado, pero, sobre todo, el respeto a la conciencia es objeto de reflexión preferente por parte de los juristas (Gratiano) y de los teólogos (San Bernardo y Pedro Lombardo). Santo Tomás de Aquino elabora el concepto de naturaleza creada, pero suficiente en cuanto a sus fines. Por último, el descubrimiento de América obliga a los teólogos españoles a sacar las consecuencias de la idea de naturaleza humana en el contexto socio-político de la Conquista.

PALABRAS-CLAVE - Filosofia Medieval. Derechos Humanos. Liberdad. Filósofos españoles.
ABSTRACT - One of the major revolutions of contemporary age in moral and legel fields is the universalization of human rights. This new reality is felt by Humanity of the end of the II Millenium as a fruit of a lengthy communal conquest in the sense that all civilizations are founded on systems of belief which intend to free Humankind from the most basic needs of human condition. Yet, occidental Cultures are specially meaningful in terms of struggle for freedom, as it is recorded in the literature of the Old and New Testament as well as in the ones of the Greeks and the Romans. Although it is not yet question of individul rights, the Chistian idea of Human dignity inspires the first attemptes to reach the upper political and legal levels in the legitimate aspiration for freedom. During the XIIth Century the first "free" towns appear, along with the move for the idea of market. Still the respect for conscience is a prevalent matter of reflection to jurists (Gratianus) and theologians (St. Bernard, Peter Lombard). St. Thomas Aquinas develops the idea of created nature, sufficient to get its objects. Man, a natural being, is entitled to behave according to his rational nature. Finally, the Discovery of America forced Spanish theologians to bring forth consequences on the idea of human dignity in the Sociopolitical frame of the Conquest.

KEY WORDS - Mediaeval Philosophy. Human Rights. Freedom. Spanish philosophers.

* Universidad de Zaragoza. 
Vivimos en la época de los Derechos Humanos. Ello constituye una de las mayores revoluciones jurídico-morales de la historia de la Humanidad. En apenas cincuenta años (1948-2000), lo que comenzó siendo un acto de protesta contra los atropellos cometidos por los totalitarismos contra la dignidad humana, se ha convertido en un movimiento universal de lucha por la promoción y dignidad de todas las personas del mundo, sin exclusión alguna. Más aún, la DUDH encierra un germen de doctrina antropológica-moral con la que se puede hacer frente a las dificultades presentes y venideras.

Aunque la DUDH refleja la mentalidad de los países occidentales en materia de concepción de la vida humana, ello no quita valor a la universalidad de sus propuestas, porque los derechos que ahí se recogen son derechos de todas las personas. De acuerdo con la Declaración, quedan eliminadas las discriminaciones por razón de nacimiento, sexo, color, país, lengua y religión. Desde el punto de vista formal, no cabe mayor universalidad. Por tanto, ampararse en la supuesta "occidentalización" de la DUDH para dejar de reconocerlos en todas las personas, es una manera arbitraria de atentar contra alguno de los tres pilares jurídicos que sustentan el concepto de persona humana: el derecho a la vida, a la libertad y a la seguridad.

No se afirma en la DUDH que éstos sean una creación de los occidentales. Al contrario, se reconoce expresamente que en todas las culturas existen sistemas de creencias que tienen por objeto liberar al hombre de las necesidades materiales y sociales. La cultura da al hombre libertad, dominio y confianza en si mismo.

Sin embargo, en razón de la manera como se ha desarrollado la cultura occidental, ha sido en ésta donde la lucha por los derechos humanos ha adquirido una primacía y singularidad que no se observa en otras culturas. Por cultura occidental entendemos la cultura que se formó en el continente europeo a partir de la fusión entre lo grecorromano, lo judio y lo germánico, y que después se difundió por otras partes de la tierra.

La visión retrospectiva hasta llegar a la DUDH de 1948 nos muestra un largo y penoso recorrido, porque ha sido infinitamente largo el periodo de tiempo en que los hombres han vivido a merced de la voluntad arbitraria del poder político y religioso. Aún hoy los derechos humanos carecen de efectividad en las tres cuartas partes del mundo.

En este recorrido, dentro de la época moderna, hay tres etapas decisivas, o tomas de conciencia colectiva, que han permanecido con valor ejemplar para la posteridad: la Bill of Rights (1689), la Declaración de independencia americana (1776), la Constitución de los Estados Unidos de América (1787) y la Declaración de los derechos del hombre y del ciudadano (1789) de la Asamblea francesa. A partir de aquí se puede hablar ya de los derechos humanos como "derechos subjetivos", derechos de la persona como valor absoluto en razón de su dignidad, igual en todos los hombres. Como se suele decir, los llustrados recogieron lo que otros habian sembrado anteriormente, posiblemente entre dolores y lágrimas. A cada uno su mérito.

En la presente comunicación vamos a exponer la aportación de algunos pensadores medievales y renacentistas a la clarificación y reconocimiento de los derechos de la persona. 
El hombre, escribe Javier Zubiri, es estructuralmente un ser moral. Dada su estructura psico-somática, con una minima actividad instintiva, el hombre se ve obligado a crear desde sí mismo el fin de sus actos. No puede vivir sin unas referencias que den sentido a su vida y a sus acciones. Esas referencias son religiosas, políticas, morales, estéticas, etc., que los propios hombres van construyendo de forma anónima y colectivamente, y trasmitiendo a otras personas. Así los restos de escritura más antiguos que poseemos, nos hablan ya de una organización política (Sumeria, 2500 a. C.) y de soluciones de problemas de convivencia expresadas en forma de sentencias (código de Hammurabi, 1500 a. C.).

La aparición de la ciudad griega (s. XII-VIII a.C.) significa un alto cualitativo en la organización de la vida y de las relaciones humanas, porque se pasa de una estructura social rígida, a causa del carácter sagrado y absoluto del soberano, a un espacio público regido por la fuerza de la razón, representada, al principio, por los "Siete sabios" (s. VIII a.C). La ciudad es asunto de todos. La justicia se desacraliza y se convierte en norma racional, sujeta a discusión.

El desarrollo de la racionalidad rompe la creencia en un orden global, y plantea de forma radical la diferencia entre la Naturaleza (physis) y la Cultura (nomos). Los Sofistas (s. V) señalan que todos los pueblos poseen una misma naturaleza física, cuyas leyes son iguales en todos (comer, beber, engendrar...) pero, al mismo tiempo, poseen leyes y costumbres diferentes de las de los griegos. Estas son variables, históricas, puras construcciones humanas, sujetas, por tanto, a crítica. La misma diferencia se observa también en los hombres individuales: por una parte están sujetos a la ley de la naturaleza física, pero, por otra, son relativamente libres, en cuanto a sus actos propiamente humanos. Decimos relativamente porque la naturaleza humana tiene su propio bien específico, y en alcanzarlo consiste la felicidad del hombre.

La aportación de Platón y, sobre todo, de Aristóteles consistió en elevar a la categoría de ciencia el conocimiento de la naturaleza humana, dando lugar a la Moral y a la Política, dos ciencias prácticas, llamadas así porque tienen por objeto la búsqueda de la racionalidad propia de la acciones del hombre como ser libre y social. Los esclavos, al no ser "libres por naturaleza", tienen únicamente carácter instrumental dentro del orden jerárquico de la Naturaleza, la cual actúa poniendo a unos al servicio de otros y así todos alcanzan su propia perfección. Es éste el lado negativo de la ética aristotélica.

La filosofía estoica sustituyó la visión particularista y etnocéntrica de las ciudades griegas (polis) por otra más universal (cosmópolis). En efecto, si la Naturaleza es igual para todos, ¿por qué no aceptar también la existencia de una ley humana universal? Lo que se gana en universalidad, se pierde en libertad individual, porque la ley humana universal participa también de los caracteres absolutos de la Naturaleza: Providencia, Destino, Solidaridad.

Entre los escritores estoicos griegos y romanos, la idea de "cosmópolis" dio lugar a reflexiones sobre la dignidad de todos los hombres, independientemente de su lengua, color y otros orígenes étnicos y culturales. Así, Cicerón (106-43 a.C.) es una pieza fundamental en el afianzamiento de la idea de dignidad de la especie humana y de cada hombre en particular. Funda el Derecho en la naturaleza humana, y elabora una doctrina sobre los "deberes" de la misma. 
El Derecho Romano recoge la idea de Ley Natural de los estoicos. Ulpiano (†223 d.C.) señala que existen derechos que no los concede la autoridad política, sino que están unidos a la propia naturaleza humana. El Derecho (ius), escribe, deriva su nombre de justicia (iustitia). Derecho es el arte de lo bueno y de lo justo (1 Inst: Dig. 1.1.1. prc.). Los preceptos del Derecho son vivir de forma honrosa y acreditada, no perjudicar a los demás y dar a cada uno lo suyo (1 reg: Dig. 1.1.10, 1).

En continuidad con la filosofía estoica, Santo Tomás admite la existencia de la "ley eterna", que se identifica con la Sabiduría divina (Sum.Th. I, q. 93. a. 1 concl.). La "Ley natural" es la presencia activa y organizada de la ley eterna en las criaturas, especialmente en el hombre. Este, debido a su razón, participa de la providencia divina de una forma activa: cuidando de sí y de otros. En efecto, la razón es la capacidad que tiene el hombre de pensar la relación entre los medios y los fines. Por eso, a diferencia de los seres irracionales, que están absolutamente determinados en cuanto a sus fines, el hombre ha recibido la capacidad de darse una ley y de conformarse a ella, discerniendo el bien y el mal (I-I, q.93.a.2.concl.). "El que la razón humana sea norma de la humana voluntad, por la que se mida su bondad, es una derivación de la ley eterna, la cual se identifica con la razón divina" (I-II, q. 94, a. 4). Santo Tomás repite aqui, casi al pie de la letra, la teoría de la "conciencia" de Cicerón (De leg., I, XIV, 40; De Repub., V, IV): el malhechor no se arrepiente del mal cometido únicamente por temor al castigo, sino por la presión de su conciencia, la cual le señala lo que hay que hacer y lo que hay que evitar, del mismo modo que la ley natural.

La ley natural es universal e inmutable (I-II, q. 94. a. 4-5). Es universal como los primeros axiomas de las ciencias, aunque hay una notable diferencia entre la razón especulativa y la razón práctica. En ambos casos los primeros principios son comunes a todos los hombres, pero, mientras que las conclusiones lógicas particulares de los principios generales de los conocimientos son verdades universales, en cambio, las aplicaciones particulares de los principios de la razón práctica pueden diferir según las circunstancias, pues puede suceder que el conocimiento de la ley natural esté falseado por la pasión o la adquisición de un mal hábito.

Así pues, el hombre ha de comenzar por asegurar el recto conocimiento de la ley natural. En el hombre, la naturaleza sola es insuficiente, y necesita la ayuda del trabajo del hombre, de la cultura. Sabemos por propia experiencia que, aunque la naturaleza nos ha dado la razón y los brazos, no podemos servirnos de ellos hasta que los hemos desarrollado con nuestro esfuerzo y experiencia. La adquisición de virtudes y de hábitos equivale a "culturizar" nuestras facultades superiores, rectificando al entendimiento y, sobre todo a la voluntad, para que quiera el objeto del entendimiento. Por otra parte, así como los primeros principios no son aún la ciencia, tampoco es suficiente la existencia de la ley natural en la conciencia para obrar bien. Hacen falta otras determinaciones. Estas se obtienen de dos maneras distintas: por derivación de preceptos particulares a partir de reglas generales, o por adaptación de esas reglas a la diversidad de las circunstancias. En el primer caso, por ejemplo, el precepto "no matar" es una conclusión derivada del principio más general "hay que hacer el bien y evitar el mal". En el segundo caso, 
en lugar de concluir a partir de un principio general, hacemos como el arquitecto que tiene que idear la forma de la casa constando con los condicionamientos del lugar y las exigencias del amo. Si se trata de aplicar una sanción, ésta vendrá determinada por la naturaleza de cada falta.

Sacar conclusiones y aportar determinaciones es la tarea propia de las "leyes humanas" o positivas. En ambos casos, las leyes humanas son derivadas de la ley natural. De acuerdo con esto, Santo Tomás afirma que el "derecho de gentes" deriva del derecho natural según el primer modo, y el "derecho civil" según el segundo (I-II, q. 95. a. 4. concl.).

La ley humana, para ser legítima, debe tener los siguientes atributos: no estar en contra de la religión, estar adaptada a la capacidad natural de aquellos a los que se va a aplicar, ser clara y general. El origen de la ley humana está en la ciudad o en su representante.

En cuanto al campo de acción que comprende la ley humana, Santo Tomás retoma la distinción de Pedro Abelardo entre crimen y pecado, entre derecho y moral, decisiva para el desarrollo de la libertad de las ideas y de las costumbres en Occidente: la ley reprime el crimen, no el castigo. En consecuencia, al Estado corresponde regular el foro externo, nunca el interno o de la conciencia, que queda reservado al individuo y a Dios (I-II, q. 78. a. 1. ad 3).

El tratamiento que da Santo Tomás al tema de la justicia, ha influido en la filosofía del derecho subyacente al derecho romano-canónico. La justicia es, ante todo, una virtud cardinal y consiste en la "voluntad permanente y constante de dar a cada uno lo que le es debido. En qué consista "lo debido", depende si se trata de justicia distributiva o conmutativa. La segunda regula los intercambios entre los individuos. Para que éstos sean justos (iguales) en cuanto al valor de lo intercambiado, Tomás establece una igualdad aritmética: a=b. Por su parte, la justicia distributiva regula la justa repartición de los bienes y de los males comunes entre los miembros de un grupo, atendiendo al lugar que cada uno ocupa dentro del mismo. Aquí la relación es de proporción:

Respecto a la propiedad de los bienes, Santo Tomás defiende el derecho natural a poseer bienes materiales en propiedad (I-II, q. 66), pero distingue entre la gestión y el uso de los bienes. Por lo mismo, la posesión no debe ser tan absoluta que no tenga en cuenta el derecho de todos a poseer, empezando por los pobres (Arist. Polit., VII, 3; San Pablo, II Tim. V, 17-187). Por eso está permitido robar en caso de necesidad, según se dice en el derecho romano: in necessitate sunt omnia communia.

Por último, Santo Tomás justifica la rebelión contra el poder político injusto, incluido el tiranicidio. Pone como condición que "el remedio no sea peor que la enfermedad", pues puede suceder que se quite a un gobernante injusto para acabar poniendo a otro igual o peor. Pero, en ningún caso compete al individuo tomar esta determinación, sino a la comunidad, que es la que entregó el poder al gobernante. Estamos ante un principio plenamente democrático: el poder político, incluido el poder real, proviene de un mandato del pueblo. Si el rey es infiel a tal mandato recibido, pierde la legitimidad del mismo. 


\section{La Escuela de Salamanca}

Santo Tomás no concreta un catálogo de derechos, pero sí expresa una determinada concepción de la persona en la que se fundan aquéllos. La base de la misma es la naturaleza humana o derecho racional, que se basa en Dios. De ahí la fórmula, frecuentemente repetida, de que "hay que obrar conforme a esa razón".

Esta tradición la desarrollan los teólogos de la llamada Escuela de Salamanca (s. XVI), destacando entre todos ellos Francisco de Vitoria, Bartolomé de Las Casas y Francisco Suárez. Parten de la tradición tomista sobre la naturaleza humana, pero a la vez integran algunos elementos del nominalismo, como la individualidad, y del humanismo, como la digninidad del hombre. Cuando apelan a la naturaleza humana como fundamento de los derechos, no la piensan de una manera abstracta y desencarnada, sino en la naturaleza de los individuos concretos. De esta forma evitan tanto el nominalismo (individualismo del singular, sin naturaleza común) como el realismo exagerado (reificación de la naturaleza humana como especie o género). Por el contrario, acentúan el equilibrio entre individualidad, sinónimo de realidad personal y de libertad, y naturaleza humana, portadora de la ley natural o derecho natural, que deriva de la luz eterna: "Lex naturalis nihil aliud est quam participatio legis aeternae in rationali creatura" (Sum. Th., I-II, q. 91, a. 2).

Aunque no hablan expresamente de la dignidad humana sino de naturaleza humana, ello es debido a que deseaban acentuar el orden divino, base de su reflexión teológico-moral, y al deseo de huir del peligro de subjetivismo, propio del protestantismo. Así pues, los derechos humanos son vistos a la luz de la naturaleza humana, y ésta a la luz de la ley etema. Esto da a los derechos humanos perennidad y eternidad.

\section{a Francisco de Vitoria (1492-1546)}

Dedicó gran atención a las cuestiones morales y políticas centrales de la época: la Reforma, el descubrimiento de América y la expansión de los negocios de los mercaderes occidentales. Sus discusiones y puntos de vista sobre estas cuestiones hicieron escuela.

En tiempo de Vitoria, la dificultad de concluir algo irrefutable en materias morales se había hecho ya evidente, tras siglos de discusión en las escuelas. ${ }^{1}$ En el campo de la moral no es posible fundar certezas apodícticas, en contra de lo que pretenderán hacer algunos filósofos posteriores (Descartes, Espinosa). Ahí radica la gran diferencia entre los juicios especulativos (explicar lo que hay) y los juicios prácticos (saber cómo hay que actuar). La verdad-falsedad afecta a ambos juicios,

Hernández, R., Los Derechos Humanos según Francisco de Vitoria, Salamanca, 1984, Castilla Urbano, F., El pensamiento de Francisco de Vitoria. Filosofia e indio americano, Anthropos, Barcelona, 1992; Pereña Vicente, L., Derechos y deberes entre indios y españoles en el Nuevo Mundo, Salamanca, 1992; Abril Castelló, V, "Los derechos humanos en el tránsito del medievo a la modernidad: la Escuela de Salamanca" en Pensamiento Medieval Hispano. Homenaje a Horacio SantiagoOtero (Coord., Soto Rábanos, J.M.A.), CSIC, Madrid. 1998, p. 512-561. Como obra de consulta, cf. Corpus Hispanorum de Pace (CHP), CSIC, Madrid, 1963-1998, 40 volúmenes de y sobre los maestros de la escuela de Salamanca. 
pero de distinta manera, pues mientras en los juicios especulativos se procede de manera apodíctica, en los juicios prácticos no es posible establecer con exactitud la verdad-falsedad; por eso, en ocasiones habrá que contentarse con la "probabilidad" moral.

El punto de referencia para conocer la corrección de nuestros actos humanos es la naturaleza humana, de la que conocemos sus fines, pero no cómo se deben realizar en la práctica. Por eso son necesarias las virtudes, en especial la prudencia.

El derecho natural consiste en obrar con rectitud natural, es decir, de acuerdo con los rasgos esenciales o constitutivos de la naturaleza humana. Estos son conocidos por las inclinaciones naturales que los protegen. Como la racionalidad es el atributo rey del hombre, ella es la que nos revela qué actos humanos están de acuerdo o en contra de tales rasgos constitutivos. El derecho natural, por tanto, es la realización de las potencialidades humanas. Actuar en contra de sus fines, es antinatural.

Características de la ley natural son la necesidad (no está sujeta a los cambios), la obligatoriedad y universalidad (hay unos mínimos que no pueden ser transgredidos sin amenazar a la persona), y la cognoscibilidad (está inscrita en el corazón de todos los hombres, incluidos "los indios, recientemente descubiertos").

Todos los hombres pueden conocer el derecho natural por ser un derecho racional. El hombre puede llegar intuitivamente al conocimiento del mismo a través del hábito natural llamado "sindéresis". Ciertos principios morales, como "buscar el bien y evitar el mal", "no cometer injusticia", etc. están en todos los hombres, y cuando desarrollamos la inteligencia, al mismo tiempo despertamos a la moralidad $\mathrm{y}$ al conocimiento de la ley natural. Esos principios no son ideas innatas ni los adquinimos por efecto social, sino que los desarrollamos con la edad.

En moral, el problema viene a la hora de determinar la operatividad de esos primeros principios en la práctica. Aquí centra Vitoria su trabajo: en señalar los mínimos que, al ser transgredidos, niegan caracteres esenciales del ser del hombre.

A partir del derecho natural, Vitoria señaló cuáles eran los derechos que acompañan a las personas en razón de su naturaleza racional:

a) Igualdad. La natura hace iguales a los hombres: "Todos los hombres eran iguales por derecho natural, y ninguno era el primero por derecho natural" (Comentarios a la "Secunda secundae" de Santo Tomás, T. III: De iustitia. Salamanca, 1934, p. 77). ${ }^{2}$

b) Libertad. Por derecho natural los hombres son libres en todos los ámbitos de la vida humana: emigración, comercio, defensa, educación, religión, etc. "Si los bárbaros permitieran a los españoles predicar el Evangelio libremente y sin obstáculo, ya reciban la fe, ya no, no es lícito por este capítulo declararles la guerra ni tampoco ocupar sus tierras" (Obras de Francisco de Vitoria, Relecciones teológicas, Madrid, 1960, p. 717). ${ }^{3}$

"[...] omnes homines de iure naturali erant aequales et nullus erat princeps de iure naturali".

"Si barbari permittant hispanos libere et sine impedimento praedicare Evangelium, sive recipiant fidem sive non, non licet hac ratione intentarte illis bellum nec alias occupare terras illorum." 
c) Vida civil y política. El hombre es un animal social por naturaleza. Por eso tiene derecho a relacionarse libremente con los demás y formar familias, ciudades, países, etc. Los gobernantes son sólo administradores del poder que les concede el pueblo.

d) Paz. El derecho a la guerra y el derecho a la paz van unidos. Por eso hay que respetar ciertas condiciones antes de declarar la guerra.

e) Derecho de los pueblos. Se plantea la colonización como un proceso temporal y con el fin de preparar a los pueblos a su autogobiemo.

f) Derecho de gentes. No sólo las personas individuales tienen derechos, sino que también los tienen todos los pueblos de la tierra, independientemente de su religión. Más aún, Vitoria plantea la teoría del Totus Orbis sobre la base del derecho natural: la existencia de una Communitas naturalis orbis, anterior y superior a todas las naciones. Todos los hombres forman parte de esta Comunidad internacional, de la que derivan derechos y obligaciones naturales. De esta forma queda sobrepasado el derecho natural y civil interno de cada Estado. "Como todo el género humano tuvo derecho a elegir un solo monarca en un principio, antes e la división de los pueblos, también podrá hacerlo ahora, puesto que ese derecho es natural y por tanto no cesa" (Obras, p. 180). ${ }^{4}$

\section{b Bartolomé de Las Casas (1484-1566)}

Intervino militarmente en la conquista de América, casi desde el principio. Como premio a su labor, recibió una "encomienda": tierra e indios para cultivarla. Al cabo de unos años volvió a la Península, recibe las órdenes sagradas y vuelve a la Isla Española (Santo Domingo), ejerciendo de sacerdote encomendero.

El sermón de fray Antón Montesinos (21 de diciembre de 1511, $4^{2}$ domingo de Adviento) ante las autoridades españolas, le abre los ojos a la realidad antihumana de la que estaba formando parte. "Ego vox clamantis in deserto: Yo soy la voz que clama en el desierto", les dijo fray Antón.

El 15 de agosto de 1514 Las Casas decide dedicarse a la defensa de los indios. En septiembre de 1522 ingresa en la Orden de Predicadores, y pasa tres años entregado a los estudios de teología y filosofía. A partir de entonces, desarrolla una intensa labor de denuncia y de promoción de los indios ante las autoridades. Entre los años 1542-1553 despliega una intensa actividad, sobre todo con la pluma. Comienza con la Brevísima relación de la destrucción de las indias (1542) y concluye con la Controversia con Juan Ginés de Sepúlveda (1550-51), entre otras obras. ${ }^{5}$ Las Controversias de Valladolid marcan, en palabras de Cervantes, tras la batalla de Lepanto, "la más alta ocasión que vieron los siglos", sólo comparable a la Declaración de los Derechos del Hombre y del Ciudadano (1792).

4 "Item, quia aliqando genus humanum habuit istam potestatem, scilicet eligendi monarcham, ut patet a principio antequam fieret divisio. Ergo et nunc potest. Cum enim illa potestas esset iuris naturalis, non cessat."

5 Beuchot, M., Los fundamentos de los derechos humanos en Bartolomé de Las Casas, Barcelona, Anthropos, 1994; Huerga, A., Fray Bartolomé de Las Casas. Obras completas. I. Vida y obras, Madrid, Alianza, 1998. 
Las Casas fundamenta los derechos humanos de los indios en la doctrina clásica del iusnaturalismo: la igualdad de naturaleza racional de todos los hombres. "Desde el principio del género humanio, todos los hombres, todas las tierras y todas las otras cosas, por el derecho natural y de gentes, fueron libres y alodiales, o sea francas o no sujetas a servidumbre. En cuanto al hombre, se demuestra porque desde el origen de la naturaleza racional todos los seres humanos nacían libres. Puesto que siendo todos los hombres de igual naturaleza, no hizo Dios a un hombre siervo, sino que a todos concedió idéntica libertad. Y la razón es que la naturaleza racional no está ordenada esencial y absolutamente a otro ser como a su fin, como de hombre a hombre, según dice Santo Tomás" (De regia potestate. Edición crítica bilingüe, dirigida por Luciano Pereña, Madrid, 1969, pp. 1617).

Respecto al origen del poder, escribe: "Ninguna sumisión, ninguna servidumbre, ninguna carga puede imponerse al pueblo sin que el pueblo, que ha de cargar con ella, dé su libre consentimiento a tal imposición. Además, los reyes, príncipes, señores y altos funcionarios que impusieron las contribuciones y tributos tuvieron su origen en el libre consentimiento del pueblo, y toda su autoridad, potestad y jurisdicción les vino a través de la voluntad popular... Luego si el pueblo fue la causa efectiva o eficiente y también la causa final de los reyes y príncipes, de forma que tuvieron su origen en el pueblo a través de elecciones libres - ut a populo per liberam electionem duxerunt -, no pudieron desde el principio imponer más tributo y servicios que los aceptados por el mueblo mismo y a cuya imposición hubiera él consentido de buena voluntad" (Ibid., p. 19).

Una nota característica de Las Casas es su discrepancia con la doctrina de Vitoria en puntos concretos.

a) Los títulos para justificar las guerras preventivas contra los indios. Sepúlveda había aprovechado la autoridad de Vitoria en este punto. Las Casas argumenta que Vitoria escribe sobre suposiciones e informes falsos. Además, escribe Las Casas, Vitoria se muestra excesivamente contemporizador para no molestar al poder. (Cf. Apología, cap. 56).

b) Derecho y deber de intervención armada que puede surgir en defensa y respaldo de la predicación. Vitoria reconoce el derecho y el deber de libre comunicación de ideas y de palabra; por eso juzga legítimo obligar por la fuerza a cumplir este derecho. Por su parte, Las Casas afirma el derecho de libertad y de autodeterminación, por el que los indios podrían cerrarse al proselitismo cristiano.

c) Atributos legítimos de los españoles para intervenir en América. Las Casas los deslegitima y exige garantías y etapas en pro del cumplimiento de todas las libertades de los indios.

En el contexto polémico en que se desenvolvió Las Casas, tal vez pueda parecer excesivo a algunos hablar de una carta lascasiana de Derechos Humanos. Efectivamente, no se puede pedir a Las Casas sistematización y tecnificación, pero sí abunda en denuncias de hechos concretos, y apunta bien en cuanto a la calificación moral y jurídica de los mismos: conculcación de la ley natural. 
Es importante recordar que, a raíz de las denuncias de Bartolomé de Las Casas y de su compañero de Orden, padre Julián Garcés (1452/60-1547), obispo de Tlaxcala, el Papa Paulo III publicó la Bula Sublimis Deus (1535), en la que denuncia que los indios sean tratados como si fueran animales salvajes. En consecuencia, manda que no sean privados de su libertad, aunque no sean cristianos. ${ }^{6}$

\section{c Francisco Suárez (1548-1617)}

Cuando Francisco Suárez llega al cenit de su saber, ha remitido la polémica americanista, pero ha surgido otra nueva, motivada por la defensa del derecho divino de los reyes, por parte de los protestantes. Un ejemplo de esta postura es la del anglicano Jacobo I de Inglaterra. Esta cuestión le llevó a Suárez a reflexionar sobre la legitimidad de la potestad civil. Siguiendo las huellas de Vitoria, Suárez defiende racionalmente el contrato social y la soberanía popular. Por haber escrito en contra del absolutismo, fue anatematizado y quemado en efigie ante los Parlamentos de Londres y de París en un solemne acto de fe inquisitorial.

La aportación de Suárez a la filosofía del derecho y de la política es tan importante o más que su contribución a la Metafísica. Pero sucedió que, para silenciar sus teorías jurídico-morales sobre el poder, Suárez fue encumbrado como metafísico.

Es característico de Suárez el carácter integrador de su doctrina: ni racionalismo objetivista ni voluntarismo subjetivista. Razón y voluntad son inseparables. Afirma que el acto creador de Dios depende de su voluntad divina, pero ésta supone el acto racional de la deliberación que sigue y determina la voluntad. De ahí que el Derecho Natural y el Derecho Positivo no existen ni operan como categorías enfrentadas y excluyentes entre sí. El Derecho Natural es tanto más Derecho cuanto más sancionado socialmente se halla en una ley o costumbre. Por la misma razón, tampoco son separables Ética y Derecho.

Comienza Suárez su obra De legibus con la definición y la división de la ley. La razón divina, en cuanto tiene razón de ley, establece reglas generales conforme a las cuales todas las cosas deben obrar. La ley eterna se limita a las criaturas racionales, y dentro de éstas a sus actos libres o morales. ${ }^{7}$

La ley natural está presente en la conciencia de los hombres. Pero no se confunde con ésta, porque la primera es una regla general constituida, y la segunda es la aplicación práctica a un caso concreto. Por eso cabe el error en la aplicación.

En lo que respecta a la libertad y autonomía de la conciencia, Suárez llega muy lejos. Pero sin caer en el subjetivismo o el laxismo, porque acepta la existencia de una moralidad objetiva.

Acerca de la sociedad, escribe que todos los hombres nacen libres, pero nos integran en una familia o sociedad determinada. Somos seres sociales por naturaleza, pero es voluntaria la constitución de la sociedad, generalmente por medio de un pacto o consenso.

\footnotetext{
6 Actas del I Congreso Intemacional sobre "Los Dominicos y el Nuevo Mundo" (Sevilla 21-15 de abril de 1987), Madrid, Edit. Deimos, 1998.

7 Suárez, F., De legibus, 8 vols., Madrid, 1960-1981. .
} 
Afirma que Dios es el origen del poder, pero el sujeto del mismo es el pueblo. Este principio de filosofía política lo elabora en el tratado De Legibus. Una vez constituida la sociedad, la autoridad dimana de ella de una manera necesaria, a la manera de una propiedad, de suerte que los particulares no pueden impedirla. Una señal de que la autoridad viene de Dios, es que a ésta corresponden actos que sobrepasan la capacidad de las personas, como dar muerte o castigar a los malhechores. Dios no entrega la autoridad mediante un acto especial distinto de la creación. "El poder político, siempre que legítima y constitucionalmente se da en un monarca o soberano, ha emanado del pueblo o comunidad, próxima o remotamente, y no puede alcanzarse de otra manera que sea justo" (De legibus, III, 4, 2).

Si los gobernantes no cumplen el compromiso contraído con los súbditos, éstos pueden desobedecerlos y desposeerlos de su cargo: "El compromiso de fidelidad $\mathrm{u}$ obediencia no obliga a veces o puede rescindirse conforme a las condiciones expresamente señaladas en el contrato constitucional entre el rey y el reino o incluidas implícitamente por exigencias del derecho natural. Y así nadie está obligado a obedecer a un rey que manda lo ilícito" (Defensio Fidei, VI, 6, 11). Si el gobernante actúa contra el bien común del pueblo o no cumple los pactos hechos con el pueblo "pueden rescindirse los pactos y deponer al rey y de este modo librarse de su obediencia y fidelidad civil" (Defensio Fidei, VI, 6, 11).

En cuanto al Derecho de gentes o derecho internacional, no lo considera basado en la ley natural, sino en una ley positivo-humana. Es un derecho no escrito, - a diferencia del derecho civil y particular -, pero está establecido entre todas o casi todas las naciones. Los preceptos del derecho de gentes son conclusiones generales que la razón hace de los principios de la ley natural, pero no por inferencia necesaria, sino en función de las necesidades, las circunstancias y la leyes civiles y privadas. La base del derecho de gentes es la tendencia natural de los hombres a unirse, como arrastrados a ello por la fuerza del amor. Todo ello va cuajando en costumbres y leyes que regulan las relaciones y los intercambios (De legibus, II, c. 19).

En este punto Suárez es menos utópico que Vitoria, quien postulaba la existencia de un imperio universal con un emperador y una superconstitución para todo el género humano. El objetivo propuesto por Suárez es más modesto: establecer las bases de una cooperación internacional, respetando la soberanía de todos los pueblos (Bonum commune omnium nationum, bonum commune generis humani. Ibid., II, c. 19, n. 9).

\section{Conclusión}

Desde la perspectiva moderna de los Derechos Humanos, se echa en falta en los autores estudiados una clara ausencia de la persona humana. Actualmente los Derechos Humanos son reinterpretados desde esta nueva visual: la persona. La persona siempre ha tenido relieve en los tratadistas cristianos, incluso entre los filósofos estoicos. Pero no es lo mismo tener presente el elemento personal, que partir de éste como criterio normativo de todo lo demás. Son dos aspectos que no son en modo alguno reiterativos. ${ }^{8}$

8 Vergés Ramírez, S., Comunicación y realización de la persona, Bilbao, Universidad de Deusto, 1987; Id., Derechos humanos fundamentales, Madrid, Tecnos, 1997. 
El camino de la fundamentación de los derechos lo han hecho por la línea de la naturaleza humana, más que de la dignidad de la persona humana. No ponen en primer lugar a la dignidad de la persona, sino el orden perenne establecido por Dios. Esto, que nos resulta chocante desde nuestra actual situación filosófica, es muy explicable desde la historia de las ideas, como hemos indicado más amiba: por una parte el horizonte teológico, centrado en el orden divino, y por otra, la explosión del subjetivismo protestante, que los teólogos católicos quieren evitar.

En un cosmos ordenado por la ley divina, el primer deber moral era aceptar el plan de Dios y tratar de cumplirlo. Dentro de este plan hay espacio para que florezcan todos los derechos humanos: igualdad, libertad, solidaridad, etc. Al razonar sobre los derechos humanos prefieren verlos a la luz de la naturaleza humana y del Dios que se identifica con la ley eterna. La inserción de los derechos humanos en esa perspectiva se produce como derechos naturales permanentes, eternos, y esa será una de las primeras formulaciones históricas, asumida en sus elementos fundamentales, más adelante, por Hugo Grocio (1583-1645). La dignidad de la persona queda en segundo lugar. Se suele decir que las doctrinas de H. Grocio, S. Pufendorf, J. Milton y L. Locke iniciaron una progresiva desteologización y secularización de los derechos naturales.

El filósofo Kant imprime un giro determinante a la fundamentación de los derechos humanos al poner la "dignidad" como piedra angular de los mismos. El hombre, escribe, es el único ser del mundo que se autoposee, en virtud de su condición racional. Por eso reserva para el hombre la palabra "dignidad". La "dignidad" es lo que le diferencia de los demás seres. Kant pone la "autonomía" como fundamento de la "dignidad", aunque, creemos, parece más justo decir que la "dignidad" se identifica con su ser racional, siendo éste el fundamento de la "dignidad" y de su "autonomía", según escribe el mismo Kant en la Fundamentación de la metafísica de las costumbres: "Obra de tal manera que trates siempre a la humanidad, ya en tu persona, ya en la de los demás, como un fin, nunca como un medio". 